\title{
Relação entre níveis de hemoglobina, concentração de retinol sérico e estado nutricional em crianças de 6 a 59 meses do Estado da Paraíba ${ }^{1}$
}

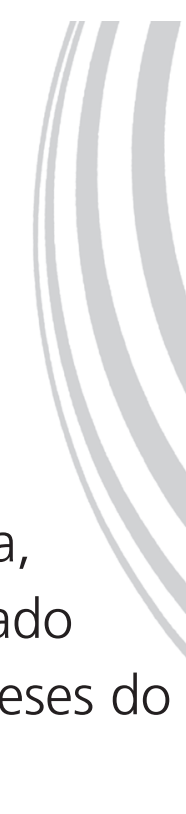

ORIGINAL | ORIGINAL

Relationship among hemoglobin levels, serum retinol

level and nutritional status in children aged 6

to 59 months from the state of Paraíba, Brazil

Sheila Sherezaide Rocha GONDIM²

Alcides da Silva DINIZ²

Mayara Poliane Pires CAGLIARI ${ }^{3}$

Emmanuele de Souza ARAÚJO3

Daiane de QUEIROZ ${ }^{3}$

Adriana de Azevedo PAIVA ${ }^{4}$

\section{R E S U M O}

\section{Objetivo}

Analisar a relação entre os níveis de hemoglobina, concentração de retinol sérico e estado nutricional em crianças de 6 a 59 meses de idade do estado da Paraíba.

\section{Métodos}

Corte transversal, de base populacional, envolvendo 1108 crianças de 6 a 59 meses de idade, de ambos os sexos, do Estado da Paraíba. As concentrações de hemoglobina foram analisadas em sangue venoso por meio decontador automático, e as de retinol sérico por cromatografia líquida de alta resolução. Para a avaliação do estado nutricional energético-proteico, foram utilizados peso e estatura pelos índices: peso/idade, estatu-

1 Artigo elaborado a partir tese de SSR GONDIM, intitulada "Magnitude, tendência temporal e fatores associados à anemia em crianças de 6 a 59 meses do Estado da Paraíba". Universidade Federal de Pernambuco; 2011. Apoio: Conselho Nacional de Desenvolvimento Científico e Tecnológico, Edital MCT/CNPq/MS-SCTIE-DECIT/SAS-DAB 51/2005 (Processo $\left.n^{\circ} 402157 / 2005-2\right)$.

2 Universidade Federal de Pernambuco, Programa de Pós-Graduação em Nutrição, Departamento de Nutrição. Av. Prof. Moraes Rego, 1235, Cidade Universitária, 50670-901, Recife, PE, Brasil. Correspondência para/Correspondence to: SSR GONDIM. E-mail: <sheilasrg@yahoo.com.br>.

${ }^{3}$ Universidade Estadual da Paraíba, Núcleo de Estudos e Pesquisas Epidemiológicas. Campina Grande, PB, Brasil.

${ }^{4}$ Universidade Federal do Piauí, Programa de Pós-Graduação em Alimentos e Nutrição, Departamento de Nutrição. Teresina, PI, Brasil. 
442 | SSR GONDIM et al.

ra/idade e peso/estatura em escores-Z. As proporções foram comparadas pelo teste do Qui-quadrado de Pearson e teste exato de Fisher, e a associação entre as concentrações de hemoglobina e de retinolemia e o estado nutricional, pelo modelo de regressão de Poisson.

\section{Resultados}

Observou-se prevalência de 36,5\% (IC95\%=33,7-39,3) de anemia (Hb 111,0 g/dL), 21,4\% (IC95\%=17,3-22,2) de hipovitaminose $\mathrm{A}(<0,70 \mu \mathrm{mol} / \mathrm{L}), 7,3 \%(\mathrm{IC} 95 \%=5,8-8,8))$ de desnutrição crônica (estatura/idade<-2), 1,3\% (IC95\%=0,6-2,0) de desnutrição global (peso/idade<-2), e 2,3\% (IC95\%=1,4-3,2) de desnutrição aguda (estatura/idade<-2). Na análise multivariada, observou-se associação direta entre anemia e retinolemia inadequada, assim como entre anemia e desnutrição crônica.

\section{Conclusão}

A elevada prevalência da anemia e da hipovitaminose A impõe a adoção de medidas efetivas de prevenção e controle. A associação entre as carências nutricionais demonstrou que uma melhoria na retinolemia bem como no estado nutricional tende a reduzir a anemia no contexto estudado.

Termos de indexação: Anemia ferropriva. Estado nutricional. Hemoglobina. Pré-escolar. Vitamina A.

\section{A B S T R A C T}

\section{Objective}

This study investigated the relationship among hemoglobin level, serum retinol level and nutritional status in children aged 6 to 59 months from the state of Paraíba, Brazil.

\section{Methods}

This was a cross-sectional, population-based study with 1,108 children of both genders aged 0659 months from the state of Paraíba. Hemoglobin ( $\mathrm{Hb}$ ) level in venous blood was determined by an automatic counter and serum retinol level by high-performance liquid chromatography. Protein-energy nutritional status was assessed by calculating the children's weight-for-age, height-for-age and weight-for-height Z-scores. Proportions were compared by the Pearson's chi-square and Fisher's exact tests and the association of hemoglobin level with retinol level and nutritional status by Poisson regression.

\section{Results}

The prevalences of anemia ( $\mathrm{Hb} \leq 11.0 \mathrm{~g} / \mathrm{dL})$ and vitamin A deficiency $(<0.70 \mu \mathrm{mol} / \mathrm{L})$ were $36.5 \%$ (C195\% 33.7-39.3) and $21.4 \%$ (CI95\% 17.3-22.2), respectively. The prevalences of chronic malnutrition (HAZ<-2), global malnutrition (weight-for-age<-2) and acute malnutrition (weight-for-height<-2) were 7.3\% (C195\% 5.8-8.8), 1.3\% (C195\% 0.6-2.0) and 2.3\% (C195\% 1.4-3.2), respectively. Multivariate analysis showed that anemia was directly associated with low serum retinol level and chronic malnutrition.

\section{Conclusion}

The high prevalences of anemia and vitamin A deficiency require the implementation of effective prevention and control measures. The association between these nutritional deficiencies demonstrated that an improvement in serum retinol levels and nutritional status tends to reduce anemia in the study context.

Indexing terms: Anemia, iron-deficiency. Nutritional status. Hemoglobins. Child, preschool. Vitamin A.

\section{N T R O D U Ç Ã O}

As condições de saúde de um indivíduo incluem como fator primordial um estado nutricional adequado baseado nos padrões de alimentação e nutrição, que são pré-requisitos indispensáveis para a promoção, proteção e recuperação da saúde'.
Ao longo das últimas décadas, o Brasil sofreu marcantes modificações políticas, econômicas e sociais que contribuíram consideravelmente para beneficiar o perfil nutricional da população infantil bem como suas condições de saúde². No entanto, as deficiências de micronutrientes ainda representam um grave problema de saúde pública. A maior proporção dessas deficiências se 
apresenta na forma subclínica, o que leva a chamar esse tipo de carência nutricional de "fome oculta" 3 .

O consumo de dietas com elevada densidade energético-proteica e deficiente em micronutrientes é comum em países industrializados e naqueles em processo de transição social e econômica, contribuindo para situações carenciais específicas ${ }^{4}$.

Diante do processo de transição nutricional no Brasil, relacionado aos aspectos econômicos, observa-se que houve uma significante mudança na situação nutricional, com redução das formas graves da desnutrição. Porém, as carências nutricionais específicas permaneceram com alta prevalência ${ }^{5}$. A deficiência de micronutrientes pode manifestar-se independente das condições socioeconômicas, e não seria determinada apenas pela falta de alimentos, como ocorre com a desnutrição energético-proteica ${ }^{6}$.

As deficiências de micronutrientes acometem aproximadamente um terço da população do mundo, causando prejuízos na saúde dos indivíduos e no desenvolvimento das nações ${ }^{7}$. O ferro e a vitamina A são os micronutrientes cujas carências apresentam as maiores prevalências no mundo ${ }^{8}$; dentre elas, a mais comum e amplamente distribuída no mundo é a deficiência de ferro, principal causa da anemia na infância7: apesar de a alta prevalência e de sua etiologia serem bem conhecidas, é um problema que persiste ${ }^{9}$ até mesmo em países desenvolvidos ${ }^{10}$.

Esse distúrbio nutricional pode provocar efeitos deletérios importantes no indivíduo. Mesmo na forma de ferropenia moderada, esse distúrbio tem sido associado à redução na capacidade produtiva, aos distúrbios do desenvolvimento cognitivo, à imunocompetência e ao desenvolvimento psicomotor ${ }^{11}$.

Por sua vez, a deficiência de vitamina A está associada com aumento acentuado da morbimortalidade, principalmente nas doenças infecciosas, e a deficiência grave pode levar a problemas visuais, inclusive a cegueira definitiva ${ }^{12}$.
As deficiências de micronutrientes muitas vezes ocorrem de forma simultânea, podendo supor que, quando uma criança apresenta a deficiência de um micronutriente, ela também estará em risco de apresentar outras deficiências concomitantes ${ }^{3}$. Existem fortes evidências de que a deficiência de vitamina A contribua para o desenvolvimento da anemia'13; por sua vez, a deficiência de ferro pode influenciar o metabolismo da vitamina A por inibir a liberação plasmática da proteína carreadora de retinol ${ }^{14}$

O sinergismo do ferro e da vitamina A não está bem elucidado, pois é complexa a interação envolvendo múltiplas vitaminas na hematopoiese. No entanto, a constatação da existência de carências nutricionais de ferro e vitamina $A$ em populações com alta prevalência de anemia ferropriva impulsiona investigações sobre a atuação desse micronutriente na gênese da anemia. Alguns achados têm demonstrado a correlação entre anemia e carência de vitamina A em crianças ${ }^{15-17}$.

No tocante à concentração de hemoglobina e ao estado nutricional em crianças, alguns achados $^{18,19}$ apresentaram correlação direta entre as duas carências nutricionais. Nesse sentido, estudos $^{1,8}$ revelaram que o estado nutricional inadequado representa um fator de risco para o desenvolvimento da anemia em crianças.

Diante da alta prevalência da anemia, da hipovitaminose $\mathrm{A}$ e da desnutrição, mesmo em patamares moderados na região Nordeste, e da provável concomitância desses distúrbios na população de risco, este estudo teve como objetivos estimar a dimensão dessas carências nutricionais e verificar a associação entre concentrações de hemoglobina e concentrações de retinol sérico e estado antropométrico em crianças de 6 a 59 meses de idade no estado da Paraíba.

\section{MÉ T O D O S}

Foi desenvolvido um estudo de corte transversal, de base populacional e domiciliar, realizado entre janeiro e abril de 2007, envolvendo crianças na faixa etária de 6 a 59 meses de idade, de ambos 
os sexos, procedentes da área urbana do estado da Paraíba. Foram consideradas elegíveis as crianças na faixa etária entre 6 e 59 meses, com residência fixa no domicílio sorteado, que não apresentasse febre, diarreia, problemas neurológicos, doenças infectocontagiosas e anormalidades físicas que comprometessem a avaliação antropométrica.

\section{Amostragem}

A amostra foi calculada estimando-se uma prevalência $(p)$ de anemia de $36,0 \%{ }^{20}$. Tomou-se como base para definição da amostra a equação ${ }^{21}$ descrita na fórmula $N=\left[E^{2} \cdot p(1-p) \cdot c\right] / A^{2}$, onde $E$ é o limite de confiança $(E=1,96)$ para uma margem de erro de $5 \%$; c é o coeficiente de correção amostral $(c=2,1)$, pois a amostra não é aleatória simples, mas por conglomerados; e $A$, a precisão aceita para a prevalência estimada $(A=4,5 \%)$. $O$ tamanho amostral mínimo obtido (n) foi de 919 crianças. No sentido de corrigir eventuais perdas e permitir uma melhor desagregação das variáveis independentes, o tamanho amostral foi corrigido por um fator proporcional de 1,25 (100/100-80), resultando em uma amostra final de 1149 crianças.

As crianças foram selecionadas segundo a técnica de amostragem do tipo múltiplas etapas. Foram estimados os dados populacionais para o ano de 2006 dos municípios do Estado, de acordo com os dados do Instituto Brasileiro de Geografia e Estatística (IBGE) 22, levantando-se o número de crianças de 6 a 59 meses de idade (15\% da população), residentes na zona urbana, com as respectivas populações acumuladas. Após o cálculo do intervalo amostral, procedeu-se à casualização dos municípios. Os municípios de João Pessoa e Campina Grande, localizados respectivamente nas mesorregiões da Zona da Mata e no Agreste, foram selecionados intencionalmente por representarem os municípios com as maiores populações do Estado. Os municípios de Patos, Belém do Brejo do Cruz, Boa Ventura, Conceição, Malta, Pedra Branca e São José de Espinharas, localizados na mesorregião do Sertão, foram selecionados de forma aleatória sistemática.

Em seguida, foram sorteados os setores censitários, o número de domicílios por cada setor, com limite máximo de 40 unidades, e por fim a unidade amostral, a fim de se avaliarem todas as crianças residentes naquele domicílio que atendessem aos critérios de elegibilidade.

No domicílio, o entrevistador realizava esclarecimentos sobre a pesquisa e solicitava a autorização do responsável pela criança para participar da pesquisa mediante a assinatura do Termo de Consentimento Livre e Esclarecido; uma vez aceita a participação, fazia-se o agendamento da criança para a realização da coleta de sangue e dos dados antropométricos (aferição do peso e da estatura) nas Unidades Básicas de Saúde da Família (UBSF).

O sangue foi coletado por punção venosa periférica. Foram coletados $4 \mathrm{~mL}$ de sangue, sendo $2 \mathrm{~mL}$ depositados em tubos com anticoagulante $K_{3}$ EDTA para análises das concentrações de hemoglobina e $2 \mathrm{~mL}$ depositados em tubos sem anticoagulante (obtenção do soro) para análise do retinol sérico. As amostras de sangue utilizadas para obtenção do soro foram depositadas em tubos protegidos da luz; após a retração do coágulo, procedeu-se à centrifugação com a finalidade de separar o soro do coágulo. Posteriormente, foi feito o acondicionamento do soro em tubos Ependorf âmbar para determinação do retinol.

As amostras de sangue e soro obtidas foram acondicionadas adequadamente e transportadas para os laboratórios de análises, mantendo-se a cadeia de frio.

\section{Análises laboratoriais}

As análises foram realizadas no Laboratório de Análises Clínicas do Hospital Universitário Lauro Wanderley da Universidade Federal da Paraíba, em João Pessoa. As concentrações de hemoglobina foram determinadas em contador automático (Sysmex SF - 3000, Roche Diagnós- 
tica). Foram consideradas anêmicas as crianças que apresentaram níveis de hemoglobina abaixo de $11,0 \mathrm{~g} / \mathrm{dL}$, considerando-se a faixa etária do estudo de acordo com a Organização Mundial da Saúde $(\mathrm{OMS})^{23}$.

A determinação do retinol sérico foi realizada no Centro de Investigação em Micronutrientes (CIMICRON), na Universidade Federal da Paraíba, João Pessoa, por meio do método de Cromatografia Líquida de Alta Eficiência (HPLC) ${ }^{24}$, considerando-se os valores estabelecidos pela OMS $^{25}$ para interpretação dos resultados encontrados do nível de retinol sérico: níveis deficientes $<0,35 \mu \mathrm{mol} / \mathrm{L}$; níveis baixos $\geq 0,35 \mu \mathrm{mol} / \mathrm{L}$ e $<0,70 \mu \mathrm{mol} / \mathrm{L}$; níveis aceitáveis $\geq 0,70 \mu \mathrm{mol} / \mathrm{L}$ e $<1,05 \mu \mathrm{mol} / \mathrm{L}$; níveis normais $\geq 1,05$.

\section{Avaliação antropométrica}

O peso e a estatura foram medidos em duplicatas, com as crianças descalças, sem acessórios na cabeça e com o mínimo de roupas. O peso foi obtido por meio da balança antropométrica digital da marca Tanita ${ }^{\circledR}$ com capacidade para $150 \mathrm{~kg}$, com precisão de $100 \mathrm{~g}$, com erro esperado de até $100 \mathrm{~g}$. O peso das crianças abaixo de 2 anos foi obtido com a pesagem da mãe ou responsável juntamente com a criança, sendo subtraído posteriormente o peso da mãe. A estatura das crianças acima de 2 anos foi verificada por meio do estadiômetro vertical da marca Seca ${ }^{\circledR}$ com escala de $0-220 \mathrm{~cm}$ e subdivisões de $0,1 \mathrm{~cm}$, fixado em parede plana sem rodapé, em ângulo de $90^{\circ} \mathrm{com}$ o chão. As crianças eram posicionadas com o corpo alinhado à parede em posição ortostática, com os pés juntos e a cabeça mantida no plano de Frankfurt. O comprimento das crianças menores de 2 anos foi verificado por estadiômetro horizontal, com erro esperado para ambos os instrumentos de até $0,2 \mathrm{~cm}$. A equipe responsável pela realização da antropometria foi previamente treinada e padronizada.

Para avaliação do estado nutricional, foram utilizados escores- $Z$ dos índices antropométricos Peso/ldade (P/I), Estatura/ldade (E/I) e Peso/Esta- tura (P/E), com pontos de cortes baseados no padrão de referência da $\mathrm{OMS}^{26}$. Sendo consideradas desnutridas as crianças com $<-2$ desvios-padrão abaixo da mediana de referência, e eutróficas as com $\geq-2$ desvio-padrão a $<2$ desvio-padrão. Foram utilizados como indicadores para a avaliação nutricional: P/I - desnutrição global; E/I - desnutrição crônica; e P/E - desnutrição aguda.

\section{Análise dos dados}

O banco de dados foi construído utilizando-se o programa Epi Info versão 6.04, em dupla entrada; em seguida, foi utilizado o modo validate para checagem de possíveis inconsistências. Posteriormente ${ }^{27}$, os dados antropométricos foram transferidos para o software WHO anthroplus software 3.0.1.

As análises estatísticas foram desenvolvidas por meio do programa estatístico Statistical Package for the Social Sciences (SPSS), versão para Windows 13.0, e Stata, versão 9.0 (Stata Corporation, College Station, EUA).

As variáveis foram testadas quanto à normalidade pelo teste de Kolmogorov-Smirnov e apresentaram distribuição normal. Na descrição das proporções, a distribuição binominal foi aproximada à distribuição normal pelo Intervalo de Confiança de 95\% (IC 95\%). As proporções foram comparadas pelo teste do Qui-quadrado de Pearson e teste exato de Ficher. A associação entre concentrações de hemoglobina e retinolemia e estado antropométrico foi testada no modelo de regres-são de Poisson.

\section{Aspectos éticos}

O projeto de investigação foi aprovado pelo comitê de ética da Universidade Estadual da Paraíba, Protocolo no 11280133000-05/2006, de acordo com as normas estabelecidas para pesquisas envolvendo seres humanos. Os pais ou responsáveis permitiram a participação da criança na 
pesquisa por meio da assinatura do Termo de Consentimento Livre e Esclarecido. Os casos diagnosticados de anemia, hipovitaminose A e desnutrição foram encaminhados às Unidades Básicas de Saúde para tratamento específico.

\section{RES U LTA DOS}

Do total de 1149 crianças previstas para o estudo, foram avaliadas 1108 em virtude da perda de $3,6 \%$ da amostra, decorrente de problemas relacionados à coleta, processamento e análise do material biológico. A prevalência de anemia ( $<11 \mathrm{~g} / \mathrm{dL}$ ) foi de $36,5 \%$ (IC 95\%=33,7 - 39,3) e a de hipovitaminose $\mathrm{A}(<0,70 \mu \mathrm{mol} / \mathrm{L})$, de $21,4 \%$ (IC 95\%=17,3 - 22,2).

No tocante ao estado nutricional, observou-se uma prevalência de desnutrição crônica (E/I) de 7,3\% (IC 95\%=5,8-8,8), de desnutrição aguda (P/E) de 1,3\% (IC 95\%=0,6-2,0) e de desnutrição global (P/I) de 2,3\% (IC 95\%=1,4-3,2).
Evidenciou-se uma maior vulnerabilidade à anemia nas crianças que apresentaram menores níveis de retinol sérico, P/l e E/l (Tabela 1). No entanto, após análise do modelo de regressão de Poisson ajustado para anemia, permaneceram significativamente associadas as variáveis retinol sérico e indicador do estado nutricional E/I (Tabela 2).

\section{I S C U S S Ã O}

Os resultados apresentados reforçam que a anemia ferropriva e a deficiência de vitamina $A$ em crianças menores de dois anos têm grande prevalência na população do Estado da Paraíba. No entanto, a desnutrição crônica apresentou cifras reduzidas, configurando o quadro da transição nutricional no País. Vale ressaltar que o problema da desnutrição energético-proteica está cedendo lugar para as elevadas carências nutricionais específicas. Esse preocupante quadro, considerando-se as consequências graves dessas

Tabela 1. Associação entre níveis de hemoglobina em crianças de 6 a 59 meses de idade, da zona urbana, e concentrações de retinol e estado antropométrico. Paraíba, 2007.

\begin{tabular}{|c|c|c|c|c|c|c|}
\hline \multirow{3}{*}{ Variáveis } & \multirow[b]{3}{*}{$n$} & \multicolumn{4}{|c|}{ Hemoglobina (g/dL) } & \multirow{3}{*}{$p$-valor } \\
\hline & & \multicolumn{2}{|c|}{$<11,0$} & \multicolumn{2}{|c|}{$\geq 11,0$} & \\
\hline & & $n$ & $\%$ & $n$ & $\%$ & \\
\hline \multicolumn{7}{|c|}{ Antropometria (escore Z) } \\
\hline \multicolumn{7}{|l|}{ Peso/Estatura } \\
\hline$<-2$ & 14 & 7 & 50,0 & 7 & 50,0 & $0,453^{*}$ \\
\hline$-2 \mid-2$ & 980 & 356 & 36,3 & 624 & 63,7 & \\
\hline$\geq 2$ & 114 & 37 & 32,5 & 77 & 67,5 & \\
\hline \multicolumn{7}{|l|}{ Estatura/ldade } \\
\hline$<-2$ & 81 & 47 & 58,0 & 34 & 42,0 & $<0,001^{*}$ \\
\hline$-2 \mid-2$ & 1008 & 345 & 34,2 & 663 & 65,8 & \\
\hline$\geq 2$ & 19 & 10 & 52,6 & 9 & 47,4 & \\
\hline \multicolumn{7}{|l|}{ Peso/ldade } \\
\hline$<-2$ & 28 & 17 & 60,7 & 11 & 39,3 & $0,012^{*}$ \\
\hline$-2 \mid-2$ & 1003 & 363 & 36,2 & 640 & 63,8 & \\
\hline$\geq 2$ & 77 & 22 & 28,6 & 55 & 71,4 & \\
\hline \multicolumn{7}{|c|}{ Retinol Sérico ( $\mu \mathrm{mol} / \mathrm{L})$} \\
\hline$\geq 0,35$ e $<0,70$ & 237 & 102 & 43,0 & 135 & 57,0 & $0,024^{* *}$ \\
\hline$\geq 0,70$ e $<1,05$ & 601 & 217 & 36,1 & 384 & 63,9 & \\
\hline$\geq 1,05$ & 270 & 83 & 30,7 & 187 & 69,3 & \\
\hline
\end{tabular}

*Teste de Qui-quadrado; ** Teste exato de Fisher. 
Tabela 2. Modelo de regressão de Poisson ajustado para anemia em crianças de 6 a 59 meses de idade. Paraíba, 2007.

\begin{tabular}{lccc}
\hline Variáveis & $\mathrm{n}$ & $\mathrm{RP}^{*}$ & $\mathrm{IC95 \%}^{* *}$ \\
\hline Antropometria (Escore-Z) & & & \\
Estatura/ldade & & & \\
$<-2$ & 34 & 1,68 & {$[1,36-2,05]$} \\
$-2 \mid-2$ & 663 & 1,00 & - \\
$\geq 2$ & 9 & 1,54 & {$[0,99-2,38]$} \\
Retinol sérico ( $\mu$ mol/L) & & & \\
$<0,35$ & 1 & 1,34 & {$[0,24-7,47]$} \\
$\geq 0,35$ e $<0,70$ & 134 & 1,38 & {$[1,10-1,74]$} \\
$\geq 0,70$ e $<1,05$ & 384 & 1,16 & {$[0,95-1,43]$} \\
$\geq 1,05$ & 187 & 1,00 & - \\
\hline
\end{tabular}

${ }^{*}$ RP: Razão de Prevalência ; **IC: Intervalo de Confiança; E/l: Estatura/ldade.

deficiências, tem mostrado que as intervenções ainda não tiveram impacto no combate às carências nutricionais, principalmente no que tange à anemia e à hipovitaminose $\mathrm{A}$.

Observa-se nessa casuística que quanto menor a retinolemia nas crianças maior a susceptibilidade à anemia, fortalecendo a hipótese de que o desenvolvimento da anemia é influenciado pela concentração de retinol. Contudo, embora sejam fortes as evidências da associação entre ferro e vitamina $A$, os mecanismos para explicá-la ainda são obscuros. Há indícios de que essa correlação deva-se ao fato de que a vitamina A beneficie a eritropoese, previna infecções ou melhore a absorção de ferro ${ }^{28,29}$. É provável, ainda, que a vitamina $A$ atue na mobilização do ferro dos estoques dos tecidos orgânicos, favorecendo a disponibilidade do ferro na hematopoiese, na síntese de hemoglobina ${ }^{13}$.

Alguns estudos têm demonstrado a correlação entre anemia e deficiência de vitamina $A$ em crianças, a exemplo daqueles verificados no Brasil $^{16}$, no Canadá $^{17}$ e na Tailândia ${ }^{15}$. Em contrapartida, Ferraz et al. ${ }^{3}$, estudando a prevalência da carência de ferro e sua associação com a deficiência de vitamina A, em Ribeirão Preto (SP), não encontrou qualquer correlação entre os níveis de retinol sérico e as concentração de hemoglobina, ferro sérico e saturação da transferrina.

Netto, et al. ${ }^{30}$ demonstram que há fortes evidências que apoiam à hipótese de uma cor- relação entre vitamina $A$ e ferro e que a suplementação de ferro associada à vitamina A melhore o estado nutricional de ferro, com reduções significativas nas prevalências de anemia. Estudo realizado com crianças paquistanesas ${ }^{31}$, utilizando suplementação de ferro, observou um aumento na concentração de retinol, indicando que o possível benefício da vitamina A no estado nutricional de ferro seria a redução na frequência de infecções, o que permite um aumento na concentração da transferrina e melhora do estado nutricional do ferro; outra hipótese para essa relação seria pela possível melhora da absorção do ferro. Garcia-Casal et al. ${ }^{32}$ reforçam essa hipótese baseados em estudo que demonstrou uma elevação na absorção de ferro não heme dos cereais em adultos anêmicos, utilizando-se doses de vitamina A ou betacaroteno. Os autores apontam que esse resultado seria decorrente da prevenção dos efeitos inibitórios dos fitatos e polifenóis na absorção do ferro.

A relação entre anemia e indicadores antropométricos ainda permanece no campo da investigação. Nessa casuística, a análise multivariada mostrou que o indicador E/l apresentou uma relação positiva com os níveis de hemoglobina. Essa associação está em concordância com vários estudos ${ }^{1,18,19}$ que constataram que a piora do estado nutricional da criança representa um fator de risco para o desenvolvimento da anemia. De Abreu et al. ${ }^{8}$, em pesquisa realizada em Caracas, 
Venezuela, encontraram valores mais baixos de hemoglobina entre o grupo dos desnutridos. Entretanto, alguns achados ${ }^{33-36}$ têm revelado que a ocorrência de anemia parece não estar associada ao estado antropométrico.

Os dados revelados nessa casuística apresentaram indícios de que a melhora na condição nutricional energético-proteica, juntamente com a correção simultânea da deficiência da vitamina A, possa ter papéis importantes no controle da anemia, principalmente em locais, a exemplo do Estado da Paraíba, que apresenta distribuição endêmica de anemia e hipovitaminose $\mathrm{A}$, embora com prevalência leve de desnutrição crônica.

\section{COLABORADORES}

SSR GONDIM participou da concepção, coleta de dados, interpretação e redação do artigo. AS DINIZ participou da concepção, interpretação, redação e revisão. MPP CAGLIARI participou da coleta de dados e revisão. ES ARAÚJO participou da coleta de dados e revisão. D QUEIROZ participou da supervisão da coleta de dados e revisão. AA PAIVA participou da elaboração e coordenação do projeto que originou o artigo, concepção e revisão.

\section{REFERÊ NCIAS}

1. Monteiro CA, Szarfarc SC, Mondini L. Tendência secular da anemia na infância na cidade de São Paulo (1984-1996). Rev Saúde Pública. 2000; 34(6): 62-2.

2. Batista Filho $M$, Rissin A. A transição nutricional no Brasil: tendências regionais e temporais. Cad Saúde Pública. 2003; 19(Supl 1):181-91.

3. Ferraz IS, Daneluzzi JC, Vannucchi H, Jordão Jr. AA, Ricco RG, Del Ciampo LA, et al. Prevalência da carência de ferro e sua associação com a deficiência de vitamina A em pré-escolares. J Pediatr. 2005; 81(2):169-74.

4. World Health Organization. Food Agriculture Organization of the United Nations. Guidelines on food fortification with micronutrients [Internet]. 2006 [cited 2007 Oct 20]. Available from: <http:// www.who.int/nutrition/publications/guide_food_ fortification_micronutrients.pdf/>.

5. Escoda MSQ. Para a crítica da transição nutricional. Ciênc e Saúde Coletiva. 2002; 7(2):219-26.
6. Ramalho RA, Saunders C. O papel da educação nutricional no combate às carências nutricionais. Rev Nutr. 2000; 13(1):11-16. doi: 10.1590/S1415-5 2732000000100002.

7. Flores H, Campos F, Araújo CRC, Underwood BA. Assessment of marginal vitamin A deficiency em Brazilian children using the relative dose response procedure. Am J Clin Nutr. 1984; 40(6):1281-9.

8. De Abreu J, Borno S, Montilla M, Dini E. Anemia y deficiência de vitamina A em ninõs evaluados em um centro de atención nutricional de Caracas. Arch Latinoam Nutr. 2005; 55(3):226-34.

9. Freire WB. La anemia por deficiência de hierro: estratégias de la OPS/OMS para combatirla. Salud Pública Méx. 1998; 40(2):199-205.

10. Neuman NA, Tanaka OY, Szarfarc SC, Guimarães PRV, Victora CG. Prevalência e fatores de risco para anemia no Sul do Brasil. Rev Saúde Pública. 2000; 34(1):57-63.

11. Brunken GS, Guimarães LV, Fisberg M. Anemia em crianças menores de 3 anos que freqüentam creches públicas em período integral. J Pediatr. 2002, 77(1):50-6.

12. Bates CJ. Vitamina A. Lancet. 1995; 345(8947): 31-5.

13. Semba RD, Bloem MW. The anemia of vitamin A deficiency: epidemiology and pathogenesis. Eur J Clin Nutr. 2002; 56(4):271-81.

14. Rosales FJ, Jang JT, Piñero DJ, Erikson KM, Beard $J \mathrm{~L}$, Ross AC. Iron deficiency in young rats alters the distribution of vitamin A between plasma and liver and between hepatic retinol and retinyl esters. J Nutr. 1999; 129(6):1223-28.

15. Bloem MW, Wedel M, Egger RJ, Speek Ajscrijver J, Saowakontha S, Schreurs WHP. Iron metabolism and vitamin A deficiency in children in Northeast Thailand. Am J Clin Nutr. 1989; 50(2):332-38.

16. Magalhães $P$, Andréa $R$, Ramalho $C C$. Deficiência de ferro e de vitamina $A$ : avaliação nutricional de pré-escolares de Viçosa (MG/Brasil). Nutrire. 2001; 21(1):41-56.

17. Willows ND, Gray-Donald K. Serum retinol is associates with hemoglobin concentration in infants who are not vitamin A deficient. Nutr Res. 2003; 23(7): 891-900.

18. Salzano AC, Torres MA, Batista Filho M, Romani SAM. Anemia em crianças de dois serviços de saúde de Recife, PE (Brasil). Rev Saúde Pública. 1985; 19(6):499-507.

19. Torres MAA, Sato K, Queiroz SS. Anemia em crianças menores de dois anos atendidas nas unidades básicas de saúde no Estado de São Paulo, Brasil. Rev Saúde Pública 1994; 28(1):290-4. 
20. Oliveira RS, Diniz AS, Benigna MJC, Silva SMM, Lola MM, Gonçalves MC, et al. Magnitude, distribuição espacial e tendência da anemia em pré-escolares da Paraíba. Rev Saúde Pública. 2002; 36(1):26-32.

21. Henderson RH, Sundaresan T. Cluster sampling to assess immunization coverage: a review of experience with a simplified sampling method. Bull World Health Organ. 1982; 60(2):253-60.

22. Instituto Brasileiro de Geografia e Estatística [Internet]. Censo Demográfico 2000 [acesso 2006 set. 20]. Disponível em: <http://www.sidra.ibge. gov.br/>.

23. World Health Organization. Iron deficiency anemia assessment, prevention, and Control: a guide for programme managers. Geneva: WHO; 2001.

24. Furr HC, Tanumihardjo SA, Olson JA. Training manual for assessing vitamin A status by use the modified relative dose response assays. Sponsored by the USAID Vitamin A field support. Washington: IVACG; 1992.

25. World Health Organization. Indicators for assessing vitamin A deficiency and their application in monitoring and evaluating interventions programs. Micronutrients series. Geneva: WHO; 1996.

26. World Health Organization. Multicentre Growth Reference Study Group standards: length/beightfor-age, wheight-for-age, wheight-for-length, weight-for-beight and body mass index-for-age. Methods and Development. Geneva: WHO; 2006.

27. World Health Organization [Internet]. Anthro 2005 software and macros [cited $2008 \mathrm{Apr} 4$ ]. Available from: <http:www.who.int/childgrowth/software/ $\mathrm{em} />$.

28. Semba RD. The role of vitamin A and related retinoids in immune function. Nutr Rev. 1998; 56(1 Pt2):S38-S48.

29. Thurnham DI, Mccabe GP, Northrop-Clewes CA, Nestel P. Effects of subclinical infection on plasma retinol concentrations and assessment of prevalence of vitamin A deficiency: metanalysis. Lancet. 2003; 362(9401):2052-8.

30. Netto MP, Priore SE, Franceschini SCC. Interação entre vitamina A e ferro em diferentes grupos populacionais. Rev Bras Saúde Matern Infant. 2007; 7(1):15-22.

31. Northrop-Clewes CA, Paracha PI, Mcloone UJ, Thurnham DI. Effect of improved vitamin A status on response to iron supplementation in Pakistani infants. Am J Clin Nutr. 1996; 64(5):694-9.

32. Garcia-Casal MN, Layrisse M. Food iron absorption: role of vitamin A. Arch Latinoam Nutr. 1998; 48(3): 191-6.

33. Almeida CAN, Ricco RG, Del Ciampo LA, Souza AM, Pinho AP, Oliveira JED. Fatores associados à anemia por deficiência de ferro em crianças préescolares brasileiras. J Pediatr. 2004; 80(3):229-34.

34. Assunção MCF, Santos IS, Barros AJD, Gigante DP, Victora CG. Anemia em menores de seis anos: estudo de base populacional em Pelotas, RS. Rev Saúde Pública 2007; 41(3):328-35.

35. Miranda AS, Franceschini SCC, Priore SE, Euclides MP, Araújo RMA, Ribeiro SMR, et al. Anemia ferropriva e estado nutricional de crianças com idade de 12 a 60 meses do município de Viçosa, MG. Rev Nutr. 2003; 16(2):163-9. doi: 10.1590/\$1415-5 2732003000200003.

36. Silva DG, Franceschini SCC, Priore SE, Ribeiro SMR, Szarfarc SC, Souza SB, et al. Anemia ferropriva em crianças de 6 a 12 meses atendidas na rede pública de saúde do município de Viçosa, Minas Gerais. Rev Nutr. 2002; 15(3):301-8. doi: 10.1590/S1415-5 2732002000300006.

Recebido em: 24/8/2011 Versão final em: 18/5/2012 Aprovado em: 5/6/2012 
